\title{
Percepção da qualidade de vida em escolares do ensino médio da cidade de Fortaleza, Ceará
}

\author{
Perception of quality of life in schools of the middle \\ school of Fortaleza City, Ceará
}

\author{
Thibério Lima de Assis ${ }^{1}$ \\ Jéssica dos Santos Martins \\ Francisco Girleudo Coutinho da Silva
}

\section{RESUMO}

Introdução: Os avanços da tecnologia estão influenciando a maneira de se comportar e de se relacionar dos indivíduos, modificando o estilo e qualidade de vida (QV) destes. Objetivo: Analisar a percepção da qualidade de vida em escolares da cidade de Fortaleza, Ceará. Métodos: Estudo de delineamento transversal e natureza quantitativa realizado com 14.348 escolares ( $53,1 \%$ do sexo masculino; $13>18$ anos), matriculados em 123 escolas da rede pública estadual de ensino da cidade de Fortaleza. Para a análise da percepção da qualidade de vida os escolares responderam a uma pergunta objetiva. Inicialmente, visando verificar se existia um relacionamento entre determinadas variáveis (sexo, faixa etária, prática de atividade física) e a percepção da qualidade de vida, recorreu-se ao teste do qui-quadrado. Todos os dados foram realizados com o auxílio do programa SPSS (Statistical Package for the Social Sciences) for Windows versão 21.0 que também auxiliou na análise descritiva. O nível de significância foi determinado em $5 \%(p<0,05)$. Resultados: Participaram do estudo 14.348 adolescentes, sendo $7.620(53,1 \%)$ do sexo feminino. A maior parte da amostra tinha idade entre 16 a 18 anos (65,0\%) e cursavam o $1^{\circ}$ ano (41,9\%). 43,7\% pertenciam ao turno da tarde, 78,7\% participavam das aulas de educação física e 79,9 \% dos adolescentes não trabalhavam. Em todos os fatores associados (sexo, idade e prática de atividade física) à percepção, a maior parte da amostra afirmou possuir uma boa qualidade de vida. Conclusão: Os resultados obtidos com a realização deste estudo permitiram inferir que, de maneira geral, não há diferença entre os gêneros, faixa etária ou prática de atividade física para percepção da QV de estudantes. Em adição, as atividades de educação em saúde no ambiente escolar são práticas de promoção da saúde indutoras de processos de transformação coletiva que incidem sobre as condições de vida da população.

\section{PALAVRAS-CHAVE:}

adolescentes; percepção; qualidade de vida.

\footnotetext{
${ }^{1}$ Faculdade Terra Nordeste - FATENE.
} 


\section{ABSTRACT}

Introduction: Advances in technology is influencing the way to behave and relate to individuals by modifying the style and quality of life (QOL) of these. Objective: To analyze the perception of quality of life in school in the city of Fortaleza, Ceará. Methods: Cross-sectional study and quantitative accomplished with students 14348 (53.1\% male; 13> 18 years) enrolled in 123 schools from public schools in the city of Fortaleza. To analyze the perception of school quality of life responded to an objective question. Initially, to identify whether there was a relationship between certain variables (gender, age, physical activity) and the perception of quality of life, we used the chi-square. All data were performed with the SPSS (Statistical Package for Social Sciences) for Windows version 21.0 also assisted in the descriptive analysis. The level of significance was determined at 5\% ( $<$ <.05). Results: The study included 14348 adolescents, and 7,620 (53.1\%) were female. The majority of the sample were between 16-18 years (65.0\%) and were enrolled in 1st year (41.9\%). 43.7\% belonged to the afternoon shift, $78.7 \%$ participated in physical education classes and $79.9 \%$ of adolescents were not working. In all associated factors (gender, age and physical activity) the perception, most of the sample claimed to have a good quality of life. Conclusion: The results of this study allowed to infer that, in general, there is no difference between genders, age or physical activity to students perception of QoL. In addition, health education activities in the school environment are health promotion practices inducing collective transformation processes that focus on the living conditions of the population.

\section{KEYWORDS:}

adolescents; perception; quality of life. 


\section{INTRODUÇÃO}

A sociedade têm diminuído de maneira significativa os níveis de atividade física, sobretudo por conta do acesso às novas tecnologias, como: internet, jogos eletrônicos, celular etc. Esses avanços da tecnologia estão influenciando a maneira de se comportar e de se relacionar dos indivíduos, modificando o estilo e qualidade de vida (QV) destes (BASTOS; SANTOS, 2010).

Por este motivo, atualmente a QV é um tema frequentemente pesquisado e nunca se falou tanto sobre sua importância. Todavia, não é fácil definir a QV, apesar da existência de muitos conceitos. O tema é tratado sob os mais diferentes olhares, seja da ciência, seja do senso comum, em abordagens individuais ou coletivas (MACEDO et al., 2015; SERRANO; LEMOS; NUNES, 2013;).

O que se sabe é que a QV dentre as diferentes classificações, pode ser afirmada como a percepção do indivíduo e de sua posição na vida, no contexto da cultura e sistema de valores nos quais vive e em relação aos seus objetivos, expectativas, padrões e preocupações (WHOQOL GROUP, 1998). Portanto, QV trata-se de um conceito bem mais amplo, que envolve a sensação de bem-estar das pessoas em seu ambiente familiar, na escola, no trabalho e no seu contexto socioeconômico e cultural.

Muito embora os hábitos e ações associados à percepção da QV, perpassam diferentes ambientes, inclusive o escolar, alguns autores defendem que a educação para a saúde tradicional nas escolas é inadequada e que mudanças no próprio contexto e organização da escola são necessárias para promover alterações de comportamentos comprometedores da saúde dos adolescentes (SIMEÃO et al. 2012).

Outrossim, como os jovens passam a maior parte do seu tempo na escola, este tem que ser considerado um cenário chave para intervenções destinadas a promover a QV dos alunos. A saber, um estudo realizado no Paraná com 608 adolescentes, identificou as variáveis associadas ao domínio psicológico da QV e os principais subgrupos associados ao risco de possuir percepção negativa da QV foram compostos por adolescentes menos ativos e que apresentavam excesso de peso, o que pode justificar o fato das recen- tes pesquisa de QV estarem sendo associadas à prática de atividade física (GORDIA et al., 2010).

Ademais, os estudos concluem a importância de se trabalhar em conexão com a sociedade e o poder público para aprimorar o conhecimento a cerca da QV e através da troca de experiências e de uma politica participativa promover uma concepção ampla e democrática de saúde e QV na adolescência. Diante do exposto o presente estudo teve como objetivo avaliar a percepção da qualidade de vida em escolares do ensino médio da cidade de Fortaleza, Ceará.

\section{METODOLOGIA}

\section{Planejamento da pesquisa}

O presente estudo é parte de um projeto maior do Grupo de Pesquisa em Atividade Física e Saúde na Escola (GPAFSE/UFC), intitulado "Associação do nível de atividade física, comportamento sedentário, duração do sono e estado de sonolência com a qualidade de vida em adolescentes do ensino público estadual da cidade de Fortaleza, Ceará".

Trata-se de um estudo de caráter transversal com natureza quantitativa e inquérito epidemiológico, realizado durante o segundo semestre letivo de 2015. Foi realizado em parceria com o laboratório do sono e ritmos biológicos da Universidade Federal do Ceará (UFC) e o programa de pós-graduação em Fisiologia do Exercício da Universidade Estadual do Ceará (UECE).

Foi realizada aplicação de questionário para análise da percepção da qualidade de vida dos indivíduos. Além disto, houve um prévio esclarecimento aos participantes sobre o desenvolvimento e objetivos da pesquisa e a participação dos escolares foi autorizada mediante assinatura do Termo de Consentimento Livre e Esclarecido (TCLE).

\section{Local do estudo}

Segundo a Secretaria de Educação do Estado do Ceará (SEDUC), existem 590 escolas de Ensino Médio no estado do Ceará e destas, 167 estão localizadas na cidade de Fortaleza. Para este estudo foram selecionadas 123 escolas, todas por regionais, representando os escolares do Ensino Médio matriculados 
nas turmas de primeiro, segundo e terceiro ano do turno manhã, tarde, noite e integral, pertencentes ao município de Fortaleza, CE. As regionais foram distribuídas conforme quadro abaixo:

Quadro 01. Recrutamento das Regionais localizadas na cidade de Fortaleza

\begin{tabular}{|l|l|}
\hline REGIONAIS & NÚMERO DE ESCOLAS \\
\hline Regional I & 19 \\
Regional II & 18 \\
Regional III & 19 \\
Regional IV & 18 \\
Regional V & 27 \\
Regional VI & 22 \\
TOTAL & 123 \\
\hline
\end{tabular}

\section{Delinemento Amostral}

Para que a estimativa do tamanho da amostra seja precisa, é necessário que a população seja suficientemente grande. Desta forma, considerando os parâmetros $p=0,5$ e erro 0,03 , segundo a Secretaria de Educação do Estado do Ceará (SEDUC), existem 590 escolas de Ensino Médio no estado do Ceará e destas, 167 estão localizadas na cidade de Fortaleza. Para este estudo foi selecionado um número de escolas que possa representar os escolares do Ensino Médio matriculados nas turmas de primeiro, segundo e terceiro ano dos turnos manhã e tarde pertencentes ao município de Fortaleza, CE. Considerando os procedimentos sugeridos por Luiz e Magnanini (2000) para o cálculo amostral em estudos epidemiológicos, foi adotado o nível de confiança de $95 \%(d p=1,96)$ e o erro amostral de 3 pontos percentuais. Além disso, como o estudo apresentou uma elevada quantidade de variáveis a serem analisadas, optou-se por determinar a proporção $50 \%$ de adolescentes com baixo nível de atividade física, a qual considera uma variância máxima. A equação utilizada para o cálculo amostral pode ser observada a seguir:

$$
\mathrm{n}=\frac{\mathrm{N} \times 1,962 \times 0,25}{1,962 \times 0,25+(\mathrm{N}-1) \times 0,032}
$$

Onde:

$\mathrm{n}=$ amostra representativa

$\mathrm{N}=$ População a ser estudada

1,96 = nível de confiança escolhido, expresso em número de desvio padrão $(d p=1,96$; intervalo de confiança de 95\%).

$0,03=$ erro amostral máximo $(3 \%)$

Desta forma, a amostra mínima de escolas foi de 120. Contudo, para corrigir o erro relacionado ao processo de seleção amostral segundo recomendações para estudos com amostragem por conglomerado (LUIZ; MAGNANINI, 2000), foi acrescentado efeito de desenho de 1,4 e margem para possíveis perdas e recusas de 30 pontos percentuais. Portanto, a amostra definitiva a ser estudada foi composta por 123 escolas.

O objetivo de fazer esta análise consiste em determinar um ponto de corte a partir do qual é possível aplicar no estudo uma amostra pertinente.

Foram excluídos do estudo os escolares com idades inferiores a 14 anos e superiores a 18 anos. Além disso, foi considerada como recusa quando o adolescente se negou a participar da coleta de dados, enquanto foi perda amostral quando o adolescente não apresentou o TCLE assinado pelos pais/responsáveis ou quando, durante a coleta dos dados, preencheu o questionário incorretamente ou desistiu da participação no estudo.

\section{Instrumentos e Procedimentos}

A coleta de dados foi realizada no período entre maio de 2015 e setembro de 2015, por uma equipe treinada, do Grupo de Pesquisa em Atividade física e Saúde na Escola (UFC/FATENE/Fanor).

\section{Percepção da qualidade de vida:}

Para avaliar a percepção da qualidade de vida, os participantes responderam a uma pergunta referente a qualidade de vida: Em geral, você diria que sua qualidade de vida é: ( ) Muito Boa, ( ) Boa, ( ) Regular ( ) Ruim ( ).

\section{Análise Estatística}

Inicialmente, visando verificar se existia um relacionamento entre determinadas variáveis (sexo, faixa 
etária, prática de atividade física) e a percepção da qualidade de vida, recorreu-se ao teste do qui-quadrado de Pearson, pois ele "detecta se existe uma associação significativa entre duas variáveis categóricas" (FIELD, 2009).

Todos os dados foram realizados com o auxílio do programa SPSS (Statistical Package for the Social Sciences) for Windows versão 21.0 que também auxiliou na análise descritiva. $O$ nível de significância foi determinado em $5 \%(p<0,05)$.

\section{RESULTADOS}

Do total de participantes 14.348 adolescentes, $53,1 \%$ eram do sexo feminino. Verificou-se que a maioria tinha idade entre 16 a 18 anos $(65,0 \%)$ e cursando o $1^{\circ}$ ano (41,9\%). 43,7\% pertenciam ao turno da tarde, 78,7\% participam das aulas de educação física e 79,9 \% dos adolescentes não trabalham.

Tabela 1. Características gerais da amostra

\begin{tabular}{llc}
\hline Variáveis & $\mathrm{N}$ & $\mathbf{\%}$ \\
\hline Sexo & & \\
Masculino & 6.728 & 46,9 \\
Feminino & 7.620 & 53,1 \\
Idade & & \\
13 a 15 & 3.824 & 26,7 \\
16 a 18 & 9.324 & 65,0 \\
$>18$ & 1.177 & 8,2 \\
Série & & \\
$1^{\circ}$ ano & 6.011 & 41,9 \\
$2^{\circ}$ ano & 5.167 & 36,0 \\
$3^{\circ}$ ano & 3.160 & 22,0 \\
Turno & & \\
Manhã & 3.350 & 23,4 \\
Tarde & 6.264 & 43,7 \\
Noite & 779 & 5,4 \\
Integral & 3.946 & 27,5 \\
Educação Física & & 78,7 \\
Sim & 11.007 & 21,3 \\
Não & 2.973 & \\
Trabaha & &
\end{tabular}

Trabalha

Sim

2.856
Não

11.384

79,9

Ao analisar a QV de acordo com o sexo, observase que tanto entre os rapazes $(55,3 \%)$ como entre as mulheres $(54,9 \%)$, o maior percentual relatou possuir uma boa QV.

Tabela 2. Qualidade de vida em relação ao sexo

\begin{tabular}{|c|c|c|c|c|}
\hline \multirow[b]{3}{*}{ QV } & \multicolumn{4}{|l|}{ SEXO } \\
\hline & \multicolumn{2}{|c|}{ Masculino } & \multicolumn{2}{|c|}{ Feminino } \\
\hline & $\mathrm{N}$ & $\%$ & $\mathrm{~N}$ & $\%$ \\
\hline Muito boa & 1.420 & 21,2 & 1.364 & 18,0 \\
\hline Boa & 3.706 & 55,3 & 4.167 & 54,9 \\
\hline Regular & 1.462 & 21,8 & 1.924 & 25,3 \\
\hline Ruim & 111 & 1,7 & 138 & 1,8 \\
\hline TOTAL & 6.699 & 100 & 7.593 & 100 \\
\hline
\end{tabular}

*QV - Qualidade de vida; Nível de significância, p<0,05

Quanto à percepção da QV relacionada à faixa etária, entre todas as idades, o maior percentual esteve direcionado aos idivíduos que afirmaram possuir uma boa QV. A saber: indivíduos com idade entre 13 e 15 anos (56,4\%); 16 e 18 anos (55,3\%) e maiores de 18 anos (49,4\%). Tabela 3 na página seguinte.

Com relação à percepção dos indivíduos que praticam ou não atividade física, os resultados são congêneres. Quanto aos que praticam atividade física, a maioria (56,5\%) classificam-se na categoria boa e nos indivíduos que não praticam, a porcentagem de indivíduos é similar (50,2\%). Tabela 4 na página seguinte.

\section{DISCUSSÃO}

Este estudo analisou a percepção da qualidade de vida dos adolescentes de escolas públicas de Fortaleza. De maneira geral, sexo, faixa etária e prática de atividade física foram potenciais fatores associados à QV.

Na caracterização dos sujeitos estudados, a maior parte da amostra era do sexo feminino $(53,1 \%)$ e a participação nas aulas de Educação Física esteve presente em uma parcela muito grande dos entrevistados (78,7\%). Caracterizá-los é necessário para o delineamento do perfil da percepção da QV dos adolescentes da cidade de Fortaleza e ao compará-lo 
Tabela 3. Qualidade de vida em relação à idade

\begin{tabular}{lllllll}
\hline & IDADE & & & & \\
QV & $13-15$ & & $16-18$ & & $>18$ & \\
& $\mathrm{~N}$ & $\%$ & $\mathrm{~N}$ & $\%$ & $\mathrm{~N}$ & $\%$ \\
\hline Muito boa & 781 & 20,5 & 1.791 & 19,3 & 210 & 17,9 \\
Boa & 2.148 & 56,4 & 5.135 & 55,3 & 578 & 49,4 \\
Regular & 837 & 22,0 & 2.190 & 23,6 & 351 & 30,0 \\
Ruim & 41 & 1,1 & 177 & 1,9 & 31 & 2,6 \\
Total & 3.807 & 100 & 9.293 & 100 & 1.170 & 100 \\
\hline
\end{tabular}

*QV - Qualidade de vida; Nível de significância, $p<0,05$

Tabela 4. Qualidade de vida em relação à prática de atividade física

\begin{tabular}{lllll}
\hline \multicolumn{5}{c}{ ATIVIDADE FÍSICA } \\
QV & $\mathrm{N}$ & $\%$ & $\mathrm{~N}$ & $\%$ \\
\hline Muito boa & 2,145 & 19,6 & 569 & 19,2 \\
Boa & 6.193 & 56,5 & 1.485 & 50,2 \\
Regular & 2.467 & 22,5 & 828 & 28,0 \\
Ruim & 164 & 1,5 & 78 & 2,6 \\
TOTAL & 10.969 & 100 & 2.960 & 100 \\
\hline
\end{tabular}

${ }^{*}$ QV - Qualidade de vida; Nível de significância, $p<0,05$

com outros estudos com inferência de outras realidades, poder além dos objetivos propostos, constatar se a realidade em que estão inseridos têm relação na percepção da QV.

O fato de grande parte da amostra revelar participar da aulas de Educação Física, gera questionamentos, uma vez que atualmente, o ser humano encontra-se cada vez mais limitado a realizar movimentos, sobretudo, devido ao avanço tecnológico. Isto é notado desde as idades mais precoces, onde crianças e adolescentes substituem atividades que envolvem esforço físico pelas novidades eletrônicas (VASCONCELLOS; ANJOS; VASCONCELLOS, 2013).

Em caráter complementar, a Educação Física Escolar tem uma relevante contribuição relacionada com o bem estar e a saúde para todos os indivíduos devendo priorizar em todos os níveis de ensino a educação para a atividade física e saúde, evidenciando as associações entre hábitos de atividade física com a melhoria da QV. Portanto, é necessário abordar a relação existente entre atividade física e QV, e a importância de sua pratica contínua em todas as faixas etárias, visando assim, conscientizar os escolares quanto a importância de se adotar um estilo de vida mais ativo fisicamente (MARANI; OLIVEIRA; GUEDES, 2007).

Diferença entre os sexos para a percepção da QV, não foram detectadas. A população masculina apresentou uma maior percepção de boa QV em comparação à população feminina, todavia, mantendo uma diferença quase irrelevante de apenas 1,6\%. Tal fato pode estar relacionado às diferenças nas condições de vida ou percepção da QV para os diferentes sexos. Alguns estudos (SULLIVAN et al., 2012) relatam QV mais baixa em mulheres, ao passo que outros não observaram diferença entre homens e mulheres (GOLD et al., 2009). Assim, o efeito do sexo sobre a QV ainda não é claro (MACEDO et al., 2015).

De todo modo, a investigação sobre a QV de determinados grupos vem crescendo em importância como mensuração avaliativa de resultados relativos aos tratamentos na área da saúde. Além das informações a cerca da QV serem de suma importância na criação de políticas públicas voltadas à saúde.

Quanto à inferência da variável faixa etária na percepção da QV, em todos os grupos etários, os indivíduos afirmaram ter uma boa percepção da QV, o que corrobora com o estudo de Matos et. al (2012) em Portugal, visto que os adolescentes independente da faixa etária e da realidade em que estão inseridos consideram ter uma boa QV. 
Sobre a prática de atividade física, $56,5 \%$ dos escolares que praticavam algum execrício físico extracurricular relataram possuir uma boa QV, o que suscita razão pois a atividade física está relacionada intrinsecamente ao estilo de vida saudável, variável que determina a QV. Todavia, o número significativo da amostra que praticam atividade física pode ser justificado por serem adolescentes em idade escolar e possuírem na própria grade curricular o exercício dentro da escola, o que em estudos transversais pode superestimar os valores descritos por eles (SOARES et al.,2011).

Este fato evidencia uma falha no reconhecimento desses indivíduos quanto ao conceito de QV. Em estudo realizado no Paraná, a prática de atividade física estave relacionada diretamente com a percepção de uma boa QV. Pode-se concluir, portanto, que indivíduos mais ativos têm uma percepção positiva em relação à QV, desta forma a variável "atividade física", surge como variável relevante no processo de percepção da QV entre adolescentes (GORDIA et al., 2010).

Diante do exposto, pode-se traçar o perfil de uma população que tem uma percepção boa da QV e que é predominantemente masculina, jovem, no início do ensino médio e que já faz parte da parcela da população economicamente ativa da sociedade, o que torna a análise de sua percepção da QV primordial, visto que inferem sobre os sujeitos estudados dois fatores estressantes importantes na influência da QV: estudo e trabalho.

Além do aspecto físico da sobrecarga causada pelo estudo concomitante com o trabalho, há também o aspecto psicológico destes indivíduos, o que influencia diretamente na percepção da QV, pois cansaço, estresse, ansiedade e depressão, são fatores preponderantes na saúde das pessoas. Não obstante, se levarmos em consideração o fato da vulnerabilidade dos adolescentes, por sua própria condição de "ser adolescente", constatamos um quadro bem mais grave na influência dos fatores. Dito de outro modo, o trabalho e estudo, nesta fase da vida, apresentam influências positivas e negativas, com maior intensidade, o que acarreta um prejuízo à saúde e consequentemente à $\mathrm{QV}$.

Ademais, as limitações desse estudo incluem um número pequeno de pesquisas, pois poucos estudos avaliam a QV de indivíduos aparentemente saudáveis, como é a proposta desta pesquisa realizada com estudantes do Ensino Médio. Além disto, estudos transversais possuem limitações metodológicas, pois não avaliam causa e efeito. Contudo, os resultados obtidos nesse estudo foram similares aos que têm sido descritos em outros estudos transversais.

Portanto, intervenções podem ser feitas no sentido de esclarecer e orientar a população sobre a prática atividade física, visto que está diretamente relacionada à QV, melhorando assim os parâmetros descritos para a saúde da população.

\section{CONCLUSÃO}

Os resultados obtidos com a realização deste estudo permitiram inferir que, de maneira geral, não há diferença entre os gêneros, faixa etária ou prática de atividade física para percepção da QV de estudantes. Em adição, as atividades de educação em saúde no ambiente escolar são práticas de promoção da saúde indutoras de processos de transformação coletiva que incidem sobre as condições de vida da população.

Diante disso, torna-se claro que a escola ao representar um importante papel na melhoria da QV, necessita de uma reformulação, incluindo a participação dos estudantes nesta construção, para a projeção de intervenções no âmbito educacional em busca de um ambiente democrático e satisfatório, influenciando portanto de maneira positiva na QV dos adolescentes.

Ainda, a realização de estudos similares com outros grupos, pemitirão o conhecimento sobre a percepção da QV em outras localidades, permitindo o acesso aos fatores ligados à QV.

\section{REFERÊNCIAS}

BASTOS, A.D.; SANTOS, A.N.A. Identificação dos níveis de qualidade de vida em escolares do município de Nossa Senhora das Dores- Sergipe. Revista Brasileira Ciência e Esporte, v.31, n.2: p. 91-103, 2010.

FIELD, A. Descobrindo a estatística usando o SPSS. 2. ed. Porto Alegre, RS: Artmed, 2009.

GOLD J.I.; MAHRER N.E.; YEE J.; PALERMO T.M. Pain, fatigue, andhealth-related quality of life in children and adolescents withchronic pain. Clin J Pain, v.25: p.407-412, 2009. 
GORDIA, A.P.; SILVA, R.C.R.; QUADROS, T.M.B.; CAMPOS, W. Variáveis comportamentais e sociodemográficas estão associadas ao domínio psicológico da qualidade de vida de adolescentes. Revista Paulista de Pediatria, v.28, n.2: p. 29-35, 2010.

LUIZ. R. R.; MAGNANINI, M. M. F. A lógica da determinação do tamanho da amostra em investigações epidemiológicas. Caderno de Saúde Coletiva, v. 8, n. 2, p. 9-28, Rio de Janeiro: ago./dez. 2000

MACEDO, R.B.; SILVA, M.J.C.; SOUSA, N.F.; SANTOS, J.V.; RODRIGUES, A.M.M.; CUMMING, S.P.; LIMA, A.V.; GONÇALVES, R.S.; MARTINS, R.A. Quality of life, school backpack weight, and nonspecific low back pain in children and adolescents. Jornal de Pediatria, v.91: p. 263-269, 2015.

MARANI, F.; OLIVEIRA, A.R.; GUEDES, D.P. Indicadores Comportamentais associados à prática de atividade física e saúde em escolares do ensino médio. Revista Brasileira Ciência e Movimento, v.15, n.2: p. 39-46, 2007.

MATOS, M.G.; GASPAR, T.; SIMÕES, C. Health-Related Quality of Life in Portuguese Children and Adolescents. Revista Psicologia: reflexão e crítica, v.25. n.2: p.230-237, 2012.

SERRANO, M.L.; LEMOS, I.; NUNES, C. Adolescent quality of olescents from the south of Portugal and Spain. Texto Contexto Enferm, v. 22, n.4: p. 893-900, 2013.

SIMEÃO, S.F.A.P.; MINATEL, F.; VITTA, A.D.; GATTI, M.A.N.; CONTI, M.H.S.D.; MARTA, S.N.; SAES, S.O. Qualidade de vida de estudantes do ensino médio. Revista Salsuvita, v.31, n.2: p. 153-168, 2012.

SOARES, A.H.R.; MARTINS, A.J.; LOPES, M.C.B.; BRITTO, J.A.A.; OLIVEIRA, C.Q.; MOREIRA, M.C.N. Qualidade de vida de crianças e adolescentes: uma revisão bibliográfica. Revista Ciência e Saúde Coletiva, v.16, n.7: p. 3197-3206, 2011.

SULLIVAN P.B.; BEALES D,J.; SMITH A.J.; STRAKER L.M. Low back painin 17 year olds has substantial impact and represents an important public health disorder: a cross-sectional study. BMC Public Health, v.12, 2012.

VASCONCELLOS, M.B.; ANJOS, L.A.; VASCONCELLOS, M.T.L. Estado nutricional e tempo de tela de escolares da Rede Pública de Ensino Fundamental de Niterói, Rio de Janeiro, Brasil. Caderno de Saúde Pública, v.29, n.4: p. 713-722, 2013.

WORLD HEALTH ORGANIZATION. Global recommendations on physical activity for health. Geneva, SW: World Health Organization, 1998. 